\title{
ENERGY EFFICIENCY IN AD HOC WIRELESS NETWORKS WITH NODE-DISJOINT PATH ROUTING
}

\author{
Smail Omar ${ }^{(1)}$, Mekkakia Zoulikha ${ }^{(2)}$, Bernard Cousin $^{(3)}$ \\ (1) Computer Science Departement, University of Mascara, Algeria. \\ (2) Computer Science Departement, University of Oran Mohamed Boudiaf-USTO, Algeria. \\ (3) IRISA / University of Rennes 1, France. \\ smailomar@gmail.com, zoulikh@gmail.com, bcousin@irisa.fr
}

\begin{abstract}
Routing in ad hoc mobile networks is a problem which is not satisfactorily solved yet. Traditional techniques of routing are not well adapted to new networks. Indeed, their lack of reactivity with respect to the traffic and network changes make them not easily usable but at the price of an oversizing of the network resources (network bandwidth, node memory utilization, node CPU load, etc). The research community was interested these last years in the improvement of the ad hoc routing, and among the solutions suggested the multipath routing has been considered. Multiple paths are exploited in order to ensure reliability, quick time reaction to changes with a low overhead generated by the control messages.

We present in this article, an extension of the well known routing protocol AODV (Ad hoc On-demand Distance Vector). We propose to improve the multipath routing strategy with a path classification enabling the choice of the paths having the best energy level.
\end{abstract}

Keywords: Computer Networks, Mobile Ad Hoc Networks, Multipath Routing, Energy Control.

\section{INTRODUCTION}

An ad hoc network is characterized by frequent changes in the network topology, the limitation on the bandwidth availability, and the limitation of the electrical power available in the network nodes. The topology of ad hoc networks frequently changes because the nodes in an ad hoc network are able to move collectively or individually in an unforeseeable manner. These characteristics make the routing complex in this type of network.

Many papers have studied routing in ad hoc networks [1, $2,3,4,5]$. Although the protocols suggested in these papers show certain relevant characteristics, they present some limits, especially when a strong mobility of the nodes or a heavy load of the network is considered.

These last years, interest in the problems of improvement of the ad hoc routing gave rise to several routing mechanisms. Among these routing mechanisms, multipath is used by several protocols to avoid useless delays during link failures [6]. These failures are sometimes due to the energy depletion of node batteries. A number of researchers have focused on the design of communication protocols that preserve energy so as to assure network service for as long as possible $[7,8,9$, 10].

The concept of the routing multipath is to give to the source node the choice, at a given moment, between multiple paths to reach a certain destination. The multiple paths can be used alternatively or in a concurrent way in accordance with some selected criteria.

In this paper, we propose a multipath routing protocol to reduce the overhead traffic and minimize the end-to-end delay of the mobile ad hoc networks.

In this paper we propose a novel on-demand routing protocol for ad hoc network based on multipath principle, in order to efficiently use energy nodes and reduce routing packets.

The remainder of the paper is organized as follows. Section 2 provides a review of related works for multipath routing in wireless ad hoc networks. Section 3 gives design details of our protocol AODVM+. Section 4 provides simulation results for performance evaluation. Section 5 concludes this paper.

\section{MULTIPATH ROUTING}

Routing multipath in ad hoc networks is a promising technique which allows to solve the problems of instability, limited band-width, conservation of energy, etc.

The use of multiple paths decreases the effect of the network link failures. This contributes highly in the improvement of the network performances. Nevertheless multipath routing remains a technique more difficult than simple routing. Its difficulty lies in the research of the (best) multiple paths.

The multipath approach makes it possible to compute multiple paths in a distributed and independent. It is based on the principle of disjunction of the multiple paths between the same source-destination pair (the disjunction can be partial [11]). The purpose of this disjunction principle is to ensure the independence of the paths, i.e. if a link of one of the paths undergone a failure, this failure will not affect the other paths.

Let us note that there are two types of path disjunction: for the links or the nodes. 


\subsection{Ad hoc On-demand Distance Vector Routing Multipath (AODVM)}

The computation of disjoint paths makes the object of several papers [12, 13, 14, 15]. For instance, AODV multipath (AODVM) is a multipath routing protocol whose objective is to find node-disjoint paths [16]. It is an extension of a single-path routing scheme known as Ad hoc On Demand Distance Vector (AODV) [17], and it allows to compute multiple loop-free and link-disjoint paths between any source and destination nodes. The propagation of RREQ (Route REQuest) follows the same rule as the basic AODV except that the intermediate nodes are disallowed to send route replies back to the source. Each node maintains a table of destinations with the corresponding path costs (hop count). To maintain this table, the source broadcasts RREQ messages. This table is called RREQ table in the following. When a RREQ message reaches the destination, a RREP (Route REPly) message is produced and returned to the last node from which the destination received the RREQ message. The RREP message contains a field indicating the identifier of the last node. The RREP message may not follow the same path than the RREQ message. Each intermediate node determines to which next node the RREP message should be sent. The routing of RREP message is based on the information recorded in the RREQ table. When an intermediate node receives a RREP message, it searches in its RREQ table a path toward the source (of the RREQ message). Then it forwards the RREP message to the next node associated to the destination in the RREQ table. In AODVM, in order to make sure that the same node does not take part in the various paths of a multiple path, when a node detects the transmission by one of its neighbors of a RREP message toward a given destination, the node removes the entry corresponding of this neighbor from its RREQ table. If an intermediate node receives a RREP message and does not find any entry for a given source (of the RREQ message), it produces a RDER message (Road Discovery Error) and forwards it to the node from which it has received the RREP message. The node, which receives a RDER message, will try to forward the RREP message to a different neighbor. These extensions solve AODV's problems. However, AODVM does not take account the battery energy of nodes, these nodes can be deleted and link failure due to the depletion of their energy.

\section{MULTIPATH ROUTING PROTOCOL AODVM+}

The aim of this article is the development of a multipath routing protocol in accordance with AODV [17] and AODVM [16] protocols. Our resulting protocol $\mathrm{AODVM}+$, is a reactive protocol for multipath routing which selects the path with the goal to save the energy of the nodes constituting the paths. AODVM+ uses the same types of message than AODVM.

\subsection{Path Discovery}

To allow the discovery of several disjoint paths between a source and a destination, instead of discarding the duplicated packets RREQ (as AODVM does), an intermediate node must proceed in the same path as first maintained RREQ message. When a node receives a duplicated RREQ message, it still establishes an reverse path towards the source by recording the address of the neighbor from which it received the first copy of RREQ message. The information contained by the RREQ message is recorded in a new field rtable_mp. The field rtable_mp represents the alternate paths, which each entry contains a list of all the paths towards the same destination and generated by the same RREQ message (all of them have the same broadcast_id), but which use different next_hop. When the RREQ message reaches the destination, we must await so that all the messages are received, if a RREQ message arrives after the timeout, then it will be ignored.

\subsection{Reverse paths}

After the reception of all RREQ messages, the destination must answer by a RREP message towards each node from which it received a RREQ message. When a node receives its first RREP message, it creates a path entry towards the node from which it received RREP message, and it updates the route_list field (an index list table of node, it is a field in the path created).

The $r p \_r o u t e \_t a b l e$ field (a list of all the paths built with this node) is created into RREP message, its value depends on the route_list field. When the node receives another RREP message, it checked if it has a path towards with the same nexthop it updates its table, if not it creates this path in the same path as the first received RREP message. Once the withdrawal period is expired, this node answers by a RREP message each node which received a RREQ in exception of the nodes by which it received a RREP, the field rp_route_table of this RREP is the concatenation of all the values of route_list of the paths in which the destination and the same one as that of first received RREP. This mechanism is repeated until the source. Each RREP now carries an additional field called sum_re_energy $y_{i}$ the sum of all residual energy of nodes in a path $i$. The re_energy ${ }_{i}^{j}$ represents the residual energy of node $j$ of path $i$. When the intermediate node receives RREP packet, its residual energy should be added with the field sum_re_energy of RREP. Before the launch of the reverse mechanism, each field sum_re_energy is initialized to zero.

After the reception of all RREP packets by the source, the latter will have all the possible paths towards the destination.

\subsection{Selection of the Alternate Paths}

At the end of the process, the source collects all the paths and removes the paths having common links and nodes. We keep only the paths with disjoined nodes. The last 
step is to classify these paths according to their energy of their nodes. In the event of rupture the best charged path (in term of residual energy) is selected, in other words, choose the path that the highest value contained in the field sum_re_energy.

When a node detects a rupture of link in a active path, these one sends a error RERR (Route ERRor) message to the node which precedes it. When a node receives a RERR message it looks for an alternate path towards the destination, if not it consults its node predecessor, this mechanism is repeated until the source, if necessary we launch a new discovery.

\section{PERFORMANCE EVALUATION OF AODVM+}

Simulation allows to test with lower costs new protocols, topologies and events which do not exist or happen. We use NS2 as a network simulator.

\subsection{Performance Parameters}

We evaluate three key performance metrics: i) Routing overhead. The routing overhead is measured as the number of control messages transmitted at each node during the simulation. Each message hop is counted as one separate transmission; ii) End-to-end delay. The endto-end delay is the transmission delay of data packets that are delivered successfully. It represents the time that the received data packets take to reach the destination from their origin; iii) Average path energy. The average energy of paths is the average energy of nodes participating in data packet transfer from the source node to the destination node.

\subsection{Performance Evaluation}

We carried out simulations to determine the effectiveness of our protocol. The principal goal of these simulations is to analyze our protocol by comparing it with other protocols, mainly AODV [17] and AODVM [16]. The values of simulation parameters are summarized in Table 1.

\begin{tabular}{|l|l|}
\hline Communication Model & Constant bit rate (CBR) \\
\hline Network Interface Type & Phy/WirelessPhy \\
\hline MAC Type & IEEE 802.11 \\
\hline Mobility Model & Random way point \\
\hline Terrain Range & $1000 \mathrm{~m} \times 1000 \mathrm{~m}$ \\
\hline Transmission Range & $250 \mathrm{~m}$ \\
\hline Number of Mobile Nodes & $20,30,40,50$ \\
\hline Data Payload & 512 bytes \\
\hline
\end{tabular}

Table 1. Simulation Parameters

In NS2 we have used the UDP/IP protocol stack: The size of the data payload is fixed at 512 bytes and the transmission frequency is 5 data packets by second. The radio model uses a shared-media radio with a nominal bit-rate of $2 \mathrm{Mb} / \mathrm{sec}$ and a nominal radio range of 250 meters.
We adopt the energy model as implemented in the NS2.33 version. We assumed that a node consumes 281.8 $\mathrm{mW}$ while receiving, and $281.8 \mathrm{~mW}$ while transmitting. The energy consumption during the idle time is not considered in this model.

In order to study the convergence of our AODVM+ protocol, we carried out a series of simulations by taking in consideration the routing overhead in the network (RREQ, RREP, RERR messages), and by changing the node density: 20, 30, 40, 50 nodes. The same scenario is applied to standard AODV protocol (see Figure 1).

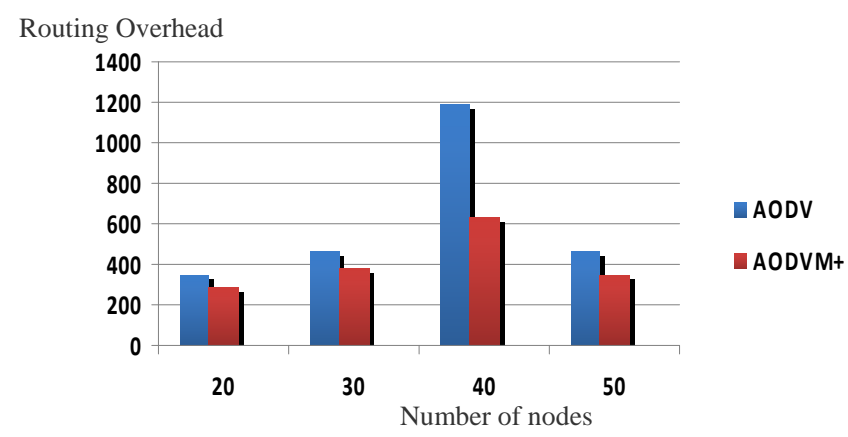

Figure 1. Routing overhead of AODV vs AODVM+

We note that the number of routing messages is more important in AODV protocol. Indeed, approximately 460 routing messages (in the case of 50 nodes) have been forwarded in the network during a $300 \mathrm{~ms}$ simulation, versus 350 for the AODVM+ protocol.

AODVM+ reduces the overhead traffic by $27 \%$.. This performance increase is provided by the use of the alternate paths when a link fails, unlike AODV protocol which starts a new path discovery (a new Request/Reply cycle), which increases the number of control packets. The second set of simulations evaluates the end-to-end delay, with the node density: 20, 30, 40, 50 nodes.

End-to-end delay (ms)

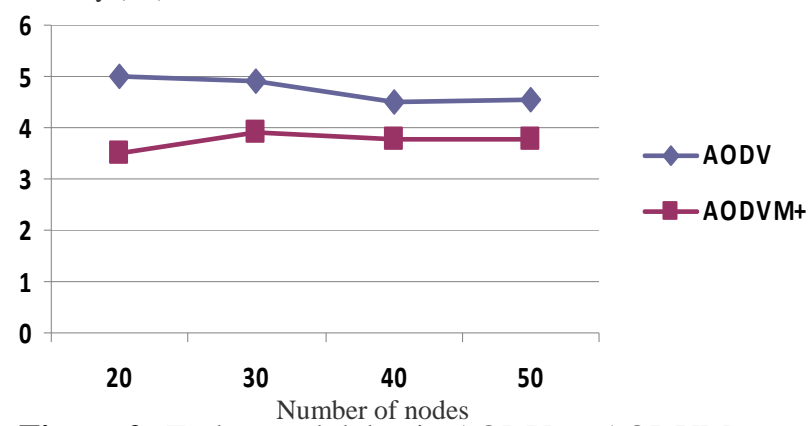

Figure 2. End-to-end delay in AODV vs AODVM+

Figure 2 shows the time taken for all packets to be transmitted across a network from source to destination. The data packet end-to-end delay is lower in our protocol (AODVM+) than in AODV protocol. We gain 22\%, this difference is the consequence of the additional cost generated by the control messages of AODV protocol. The last set of simulations is related to the quality of the alternate paths based on the residual energy of the path nodes. 
Our contribution lies in the classification of the paths by their residual energies. We evaluate the two protocols AODVM and AODVM+.

Average Path Energy

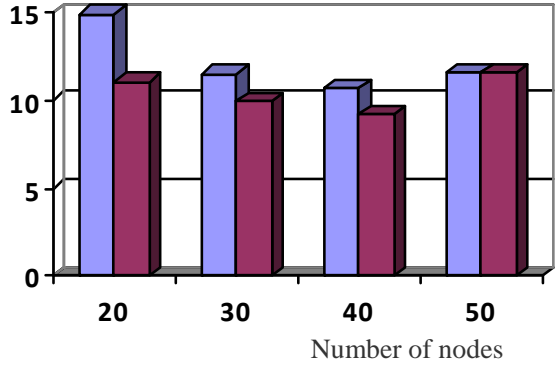

$\square$ AODVM+

$\square$ AODVM

Figure 3. Average energy of paths in AODVM vs AODVM+

Figure 3 shows that the average residual node energy of the paths discovered in our protocol AODVM+ is greater than in protocol AODVM. That means that our protocol uppermost uses the most loaded paths (in terms of node battery energy), this ensures the lifetime of the network and contributes considerably to its reliability.

\section{CONCLUSION}

Through this article we have brought a solution to the problems involved by link failures due to node mobility in ad hoc network routing. Our approach consists ina multipath extension to the AODV routing protocol.

The proposed multipath approach allows us to initiate path discovery during less frequent discovery process, minimizing the overhead traffic and reducing the end-toend delay. When there is a link failure, alternative paths are available and used to forward the data packets. Discovery process is initiated only after exhaustion of all the alternate paths.

This work shows some interesting prospects because our AODVM+ solution can also be extended to include some criteria of security and energy management during the selection of the alternate paths.

\section{REFERENCES}

[1] R.D. Samir, E.P. Charles, M.R. Elizabeth, "Performance comparison of two on-demand routing protocols for ad hoc networks,” IEEE Conference on Computer Communications, (INFOCOM 2000), September 2000.

[2] A. Cedric, C. Thomas, J. Philippe, L. Anis, M. Pascale, M. Paul, Q. Amir, and V. Laurent, "Optimized Link State Routing Protocol,” IETF RFC 3626, October 2003.

[3] D. Johnson, D. Maltz, "Dynamic source routing in ad-hoc wireless networks," Computer Communication Review, in Proc. of SIGCOMM 96, August 1996.

[4] D. Murthy, J.J. Garcia-Luna-Aceves, "A Routing Protocol for Packet Radio Networks,” 1st ACM Int'l Conference on Mobile Computing and Networking (Mobicom'95), November 1995.

[5] V.D. Park, M.S. Corson, "A Highly Adaptive Distributed Routing Algorithm for Mobile Wireless Networks," IEEE conference on Computer Communications (INFOCOM 97), April 1997.

[6] D. G. S. Mueller, "Multipath routing in mobile ad hoc networks: issues and challenges," Performance Tools and Application to Networked Systems, Lecture Notes in Computer Science, vol. 2965, April 2004.

[7] S. Singh, M. Woo, C.S. Raghavendra,"Power-aware routing in mobile ad hoc networks," ACM/IEEE Mobicom. October 1998.

[8] C.-K. Toh, "Maximum battery life routing to support ubiquitous mobile computing in wireless ad hoc networks," IEEE Communications Magazine 39 (6), pp.138-147, June 2001.

[9] L. Y. Li, F. Zheng, C. L. Li and P. Y. Yuan, "An Energy Constrained Routing Protocol for MANET", Int. Conference on Machine Learning and Cybernetics, Hong Kong, China, Vol. 6. August 2007.

[10] A. Aksu and O. Ercetin, "Reliable Multi-hop Routing with Cooperative Transmissions in Energy-Constrained Networks", IEEE Trans. on Wireless Commun., Vol. 7, No. 8, pp: 28612865, August 2008.

[11] W. Lou, W. Liu, Y. Zhang, "Performance Optimization using Multipath Routing in Mobile Ad Hoc and Wireless Sensor Networks,” Combinatorial Optimization In Communication Networks. Kluwer Academic Publishers. 2005.

[12] M. K. Marina and S. R. Das, "On-Demand MultiPath Distance Vector Routing in Ad hoc Networks", in Proc. of the Ninth International Conference on Network Protocols (ICNP), California, IEEE Computer Society Press, pp. 14-23, November 2001.

[13] R. Leung, J. L Liu, E. Poon, A. C. Chan, B. Li, "MPDSR: A QoS Aware Multi-path Dynamic Source Routing Protocol for Wireless Ad hoc Network," In Proc. 26th Annual IEEE International Conference on Local Computer Networks (LCN 2001), pp. 132-141, November 2001.

[14] G. Parissidis, V. Lenders, M. May, B. Plattner, "Multipath Routing Protocols in Wireless Mobile Ad Hoc Networks: A Quantitative Comparison,” Lecture Notes in Computer Science, Vol. 4003, pp. 313-326, September 2006.

[15] X. Li, L. Cuthbert, "Node-Disjointness based Multipath Routing for Mobile Ad hoc Networks", in Proc. of the ACM International Workshop on Performance Evaluation of Wireless Ad-hoc, Sensor and Ubiquitous Networks, ACM Press, pp. 2329, October 2004.

[16] Z. Ye, S.V. Krishnamurthy, S.K.Tripathi, “A framework for reliable routing in mobile ad hoc networks," IEEE (INFOCOM 2003), March 2003.

[17] C.E. Perkins and E.M. Royer, "Ad hoc on-demand distance vector routing," in Proc. of the IEEE Workshop on Mobile Computing Systems and Applications (WMCSA), pp. 90-100, February 1999. 